\title{
Role of transvaginal sonography in gynecological diagnosis at a tertiary care hospital
}

\author{
Sitalakshmi V. ${ }^{*}$, Alpana Bansal ${ }^{2}$
}

\begin{abstract}
${ }^{1}$ Department of Obstetrics and Gynecology, Narayana Medical College, Nellore, Andhra Pradesh, India ${ }^{2}$ Department of Obstetrics and Gynecology, Late Baliram Kashyap Memorial Government Medical College, Jagdalpur, Chhattisgarh, India
\end{abstract}

Received: 27 March 2017

Accepted: 26 April 2017

\author{
*Correspondence: \\ Dr. Sitalakshmi V., \\ E-mail: drvsitalakshmi@gmail.com
}

Copyright: (c) the author(s), publisher and licensee Medip Academy. This is an open-access article distributed under the terms of the Creative Commons Attribution Non-Commercial License, which permits unrestricted non-commercial use, distribution, and reproduction in any medium, provided the original work is properly cited.

\begin{abstract}
Background: Transvaginal sonography (TVS) is very useful to assess the abnormalities in the anatomical structure of the uterus as well as endometrium. Not only this, it is capable of finding out any abnormalities of ovary, fallopian tubes, cervical structures as well as myometrium. The objective of the study was the role of transvaginal sonography in gynecological diagnosis at a tertiary care hospital.

Methods: Present hospital based prospective study was carried out among 31 randomly selected eligible patients as per inclusion and exclusion criteria of the study for a period of one year at Department of Obstetrics and Gynecology of S. V. Medical College, Tirupati. All ethical guidelines were followed. A pre-designed and semi structured pretested questionnaire was prepared for entry of patient data.

Results: Transvaginal sonography was more accurate in terms of evaluation of both tubes. Overall diagnostic accuracy of transvaginal sonography was $90.9 \%$. It was found that the accuracy of clinical diagnosis was $100 \%$ in comparison to diagnosis by scan. In five cases, the clinical diagnosis of infertility was made as ovarian cyst and in all those cases it was confirmed. In three cases, the clinical diagnosis of infertility was made as fibroids and in all those cases it was confirmed. It was found that in cases of ectopic pregnancy, copper T missing loop and missed abortion, the clinical diagnosis was $100 \%$ accurate in comparison to scan diagnosis. Only in case of pelvic inflammatory disease, the accuracy of clinical diagnosis was $66 \%$ in comparison to scan diagnosis.

Conclusions: Despite the few disadvantages of transvaginal sonography in measuring large pelvic masses, the smallest details of the masses yield valuable preoperative information. The more accurate delineation of internal echo characteristics scores the benefit over transvaginal sonography has its rightful place in gynecological practice has diagnostic and minimally invasive therapeutic tool.
\end{abstract}

Keywords: Assessment, Endometrium, Hyperplasia, Transvaginal sonography

\section{INTRODUCTION}

Transvaginal sonography (TVS) is an important tool in the diagnosis of gynecological disorders especially in the diagnosis of abnormal uterine bleeding. Inspite of this, its role in the diseases of endometrial cavity has been speculated as doubtful. If one gets, on doing transvaginal sonography, the finding as thickened central endometrial complex, then it is considered as non-specific. This is considered as due to presence of submucosal fibroids, endometrial polyp, malignancy, hyperplasia of endometrium, or due to the presence of cystic atrophy. 
One more limitation of transvaginal sonography is its limitation in the diagnosis of focal lesions. This is due to its inability to evaluate double layer thickness. Inspite of these limitations, transvaginal sonography is considered as a very good diagnostic method. ${ }^{1}$ For evaluation of gynecological disorders, in addition to use of transvaginal sonography, detailed history and complete and thorough clinical examination is very important. Specific history related to menstrual cycle in relation to frequency, regularity, predictability, the amount of bleeding, duration of bleeding and character of bleeding should be carefully noted. This should not be preceded by any recognizable or consistent pattern of pre-menstrual molimina and should not be accompanied by any abnormality of the genital tract. ${ }^{2}$ Abnormal vaginal bleeding is one of the most common presenting complaints in women of any age seeking gynecologic health care. Two of the most frequently used diagnostic tests to investigate the cause of the bleeding are endometrial biopsy and transvaginal sonography. The most worrisome cause of abnormal bleeding is endometrial carcinoma, yet benign etiologies are far more prevalent, including fibroids, polyps, and endometrial atrophy. Endometrial biopsy and transvaginal sonography have equal sensitivities for carcinoma, but sonography is far more effective in diagnosing benign disease. ${ }^{3}$

In the women, about $10 \%$ have an endometrial cancer and an additional $20 \%$ have some other endometrial abnormality. However, some abnormalities, such as endometrial polyps and submucous fibroids, are difficult to diagnose by dilatation and curettage. In such cases, combining transvaginal sonography with hysteroscopy may be of value. ${ }^{4}$ Sonohysterography has become the standard test in the evaluation of dysfunctional uterine and postmenopausal bleeding because it allows reliable differentiation between focal and diffuse endometrial and subendometrial lesions, with the most common being polyps and submucosal fibroids. The major advantage of sonohysterography is that it can accurately depict the percentage of the fibroid that projects into the endometrial cavity. ${ }^{5}$ Hence present study was planned to evaluate the role of transvaginal sonography in the diagnosis of gynecological diseases.

\section{METHODS}

Present study was hospital based prospective study carried out at department of Obstetrics and Gynecology of S. V. Medical College, Tirupati. Study was conducted from March 2013 to February 2014, for a period of one year. Patients presenting with gynecological diseases were included in the study. Thus, during study period, it was possible to include 31 patients in the study. 31 patients were selected randomly during the study period

\section{Inclusion criteria}

- $\quad$ Patients presenting to gynecology department during the study period with some gynecological disease

- Patients willing to participate in the study.

\section{Exclusion criteria}

- Patients with other serious conditions were excluded from the study

- Patients not giving consent to participate in the study were not included

- Bed ridden patients were also not included in the study.

Present hospital based prospective study was carried out among 31 randomly selected eligible patients as per inclusion and exclusion criteria of the study for a period of one year at Department of Obstetrics and Gynecology of S. V. Medical College, Tirupati. All ethical guidelines were followed. A pre-designed and semi structured pretested questionnaire was prepared for entry of patient data. Detailed history and thorough clinical examination was carried out for all patients. Based on history and clinical examination findings, a clinical diagnosis was made. All patients participating in the study were referred for transvaginal sonography in the radiology department with due instructions.

They were asked to come and report to the department of Obstetrics and Gynecology. Upon arrival of the patients in the department, their report details were entered in the questionnaire. Based on the diagnosis, the patients were treated or subjected for further evaluation and complete management. The data was entered and analyzed using proportions.

\section{RESULTS}

Out of total 31 cases selected for the present study, tubal factor evaluation was done in 22 cases and evaluation of infertility was done in nine cases by transvaginal sonography.

Table 1: Tubal factor evaluation by transvaginal sonography.

\begin{tabular}{|c|c|c|c|c|c|}
\hline \multirow{2}{*}{ Tubal factor } & \multicolumn{2}{|c|}{ Diagnosis by TVS } & \multicolumn{2}{|c|}{ Diagnosis by laparotomy } & \multirow[t]{2}{*}{ Diagnostic accuracy \% } \\
\hline & No. & $\%$ & No. & $\%$ & \\
\hline Both tubes patent & 12 & 54.5 & 12 & 54.5 & 100 \\
\hline Single tube block & 04 & 18.2 & 02 & 09.1 & 50 \\
\hline Both tubes block & 06 & 27.3 & 06 & 27.3 & 100 \\
\hline Total & 22 & 100 & 20 & 100 & 90.9 \\
\hline
\end{tabular}


Tubal factor evaluation was done in a total of 22 cases out of 31 cases. The diagnosis done by transvaginal sonography was later confirmed by laparotomy. It was found that in case of both tubes patent or blocked; the accuracy of transvaginal sonography was $100 \%$. But in case of single tube evaluation, the diagnostic accuracy of transvaginal sonography was found to be only $50 \%$. Thus, we can say that transvaginal sonography was more accurate in terms of evaluation of both tubes. Overall diagnostic accuracy of transvaginal sonography was $90.9 \%$.

Table 2: Transvaginal sonography in infertility.

\begin{tabular}{|llllll|}
\hline Clinical complaint & Number of cases & Clinical diagnosis & Scan diagnosis & Confirmation & Remarks \\
\hline Anxious to conceive & 05 & Ovarian cyst & Ovarian cyst & Confirmed & $100 \%$ \\
\hline Anxious to conceive & 03 & Fibroids & Fibroids & Confirmed & $100 \%$ \\
\hline
\end{tabular}

It was found that the accuracy of clinical diagnosis was $100 \%$ in comparison to diagnosis by scan. In five cases, the clinical diagnosis of infertility was made as ovarian cyst and in all those cases it was confirmed. In three cases, the clinical diagnosis of infertility was made as fibroids and in all those cases it was confirmed.
It was found that in cases of ectopic pregnancy, copper $\mathrm{T}$ missing loop and missed abortion, the clinical diagnosis was $100 \%$ accurate in comparison to scan diagnosis. Only in case of pelvic inflammatory disease, the accuracy of clinical diagnosis was $66 \%$ in comparison to scan diagnosis.

Table 3: Clinical parameters comparing with sonographic parameters $(\mathbf{N}=\mathbf{2 8})$.

\begin{tabular}{|lllll|}
\hline Clinical diagnosis & Number of cases & Scan diagnosis & Confirmation & Percentage \\
\hline Ectopic pregnancy & 06 & 06 & 06 & 100 \\
\hline Copper T missing loop & 04 & 04 & 04 & 100 \\
\hline Pelvic inflammatory disease & 06 & 04 & 04 & 66 \\
\hline Missed abortion & 03 & 03 & 03 & 100 \\
\hline
\end{tabular}

\section{DISCUSSION}

Tubal factor evaluation was done in a total of 22 cases out of 31 cases. The diagnosis done by transvaginal sonography was later confirmed by laparotomy. It was found that in case of both tubes patent or blocked; the accuracy of transvaginal sonography was $100 \%$. But in case of single tube evaluation, the diagnostic accuracy of transvaginal sonography was found to be only $50 \%$. Thus, we can say that transvaginal sonography was more accurate in terms of evaluation of both tubes. Overall diagnostic accuracy of transvaginal sonography was $90.9 \%$. It was found that the accuracy of clinical diagnosis was $100 \%$ in comparison to diagnosis by scan. In five cases, the clinical diagnosis of infertility was made as ovarian cyst and in all those cases it was confirmed. In three cases, the clinical diagnosis of infertility was made as fibroids and in all those cases it was confirmed. It was found that in cases of ectopic pregnancy, copper $\mathrm{T}$ missing loop and missed abortion, the clinical diagnosis was $100 \%$ accurate in comparison to scan diagnosis. Only in case of pelvic inflammatory disease, the accuracy of clinical diagnosis was $66 \%$ in comparison to scan diagnosis.
Goyal BK et al found that menorrhagia was the commonest presenting symptom in the study population ( $n=58$ ) followed by metrorrhagia, menometrorrhagia and continuous bleeding $>21$ days. $^{2} 74$ female patients had normal size uterus. In 57 patients, the uterine cavity was normal on TVS. Thickened endometrium, endometrial polyp and submucous fibroids were seen in 19, 16 and 6 patients respectively. Hysteroscopy showed normal cavity in 59 female patients and polypoidal endometrium, polyps or submucous fibroids in 41. TVS was found to have high sensitivity and specificity (95.23 and 94.82 respectively) and high positive and negative predictive value. Strength of agreement between TVS and hysteroscopy was high (kappa value 0.898).

Bree RL et al concluded that HSG and transvaginal US in patients with PMB improves diagnostic accuracy, clinical decision making, and the clinician's diagnostic certainty. ${ }^{6}$ In patients with benign causes of PMB, the absence of abnormality at HSG and a normal endometrial biopsy result may eliminate the need for further studies. Dubinsky TJ et al observed that 259 patients who underwent TVS, 57 patients who had an endometrium thicker than $5 \mathrm{~mm}$ and an endoluminal mass on 
hysterosonography had false-negative results on aspiration biopsies. $^{7}$ Of the 18 patients who had malignancies in this series, 12 had false-negative results on biopsies. In the 94 patients with an enlarged uterus and negative EMB results who underwent hysterectomy, we found 87 with fibroids, three with adenomyosis, and four with sarcomas. Of the 64 women with endometria thinner than $5 \mathrm{~mm}$ seen on TVS, 21 had negative results from dilatation and curettage.

Abu-Ghazzeh $\mathrm{Y}$ et al concluded that the combination of transvaginal sonography and transvaginal hysterosonography is both sensitive and specific with regard to detecting and excluding endoluminal masses as the cause of post-menopausal bleeding. ${ }^{8}$ Diagnostic dilatation and curettage fails to detect a large percentage of some lesions, so TVS in combination with TVHS should be considered as the initial examination in the evaluation of all women with post-menopausal bleeding. O'Connell LP et al noted that the combination of endometrial biopsy and transvaginal sonohysterography positively correlated with the surgical findings $>95 \%$ of the time, with a sensitivity and specificity of $94 \%$ and $96 \%$, respectively (confidence interval $91 \%$ to $99 \%$ ). ${ }^{9}$ No patients with endometrial hyperplasia or cancer were misdiagnosed.

Holst J et al carried out a retrospective study on 2018 Scandinavian women undergoing conventional and aspiration curettage was performed. ${ }^{10}$ The outcome in terms of endometrial pathology was analyzed against age and indications. A large number of operations $(38.2 \%)$ were performed on young women before the age of 45 years. $98 \%$ of endometrial samples were normal from 430 younger than 40 years. Better selection of cases might help to reduce the number of operations. In women of reproductive age the relation between normal and pathological findings was the same for both methods with the exception that conventional curettage seemed to detect more polyps. The frequency of insufficient samples after aspiration curettage increased markedly with age. Postmenopausal bleeding remains a strong indication for conventional curettage.

\section{CONCLUSION}

Despite the few disadvantages of transvaginal sonography in measuring large pelvic masses, the smallest details of the masses yield valuable preoperative information. The more accurate delineation of internal echo characteristics scores the benefit over transvaginal sonography has its rightful place in gynecological practice has diagnostic and minimally invasive therapeutic tool.

Funding: No funding sources Conflict of interest: None declared
Ethical approval: The study was approved by the Institutional Ethics Committee

\section{REFERENCES}

1. Aslam M, Ijaz L, Tariq S. Comparison of Transvaginal Sonography and Saline Contrast Sonohysterography in Women with Abnormal Uterine Bleeding: Correlation with Hysteroscopy and Histopathology. Int $\mathbf{J}$ Health Sci (Qassim). 2007;1(1):17-24.

2. Goyal BK, Gaur I, Sharma S. Transvaginal sonography versus hysteroscopy in evaluation of abnormal uterine bleeding. Med J Armed Forces India. 2015;71(2):120-5.

3. Bubinsky TJ. Value of sonography in the diagnosis of abnormal vaginal bleeding. J Clin Ultrasound. 2004;32(7):348-53.

4. Karlsson B, Granberg S, Hellberg P. Comparative study of transvaginal sonography and hysteroscopy for the detection of pathologic endometrial lesions in women with postmenopausal bleeding. J Ultrasound Med. 1994;13(10):757-62.

5. Davis PC, O'Neill MJ, Yoder IC. Sonohysterographic findings of endometrial and subendometrial conditions. Radiographics. 2002;22(4):803-16.

6. Bree RL, Bowerman RA, Bohm-Velez M. US evaluation of the uterus in patients with postmenopausal bleeding: A positive effect on diagnostic decision making. Radiol. 2000;216(1):260-4.

7. Dubinsky TJ, Parvey HR, Maklad N. The role of transvaginal sonography and endometrial biopsy in the evaluation of peri- and postmenopausal bleeding. AJR Am J Roentgenol. 1997;169(1):145-9.

8. Abu-Ghazzeh Y, Shakoury WA, Barqawi R. Comparative study of transvaginal hysterosonography and biopsy for the evaluation of post-menopausal bleeding. Ann Saudi Med. 1999;19(2):116-9.

9. O’Connell LP, Fries MH, Zeringue E. Triage of abnormal postmenopausal bleeding: a comparison of endometrial biopsy and transvaginal sonohysterography versus fractional curettage with hysteroscopy. Am J Obstet Gynecol. 1998;178(5):956-61.

10. Holst J, Koskela O, von Schoultz B. Endometrial findings following curettage in 2018 women according to age and indications. Ann Chir Gynecol. 1983;72(5):274-7.

Cite this article as: Sitalakshmi V, Bansal A. Role of transvaginal sonography in gynecological diagnosis at a tertiary care hospital. Int J Reprod Contracept Obstet Gynecol 2017;6:2910-3. 